\title{
Combination of Texture and Shape Features Using Machine and Deep Learning Algorithms for Breast Cancer Diagnosis
}

Norhene Gargouri ( $\square$ norhene.gargouri@gmail.com )

Centre de Recherche en Numerique de Sfax https://orcid.org/0000-0003-1613-2115

Raouia Mokni

College of computer engenieering and science, Prince Sattam Bin Adulaziz University

Alima Damak

Ecole Nationale d'Ingénieurs de Sfax: Ecole Nationale d'Ingenieurs de Sfax

Dorra Sellami

Ecole Nationale d'Ingénieurs de Sfax: Ecole Nationale d'Ingenieurs de Sfax

\section{Riadh Abid}

Al-Farabi Radiolgy center

\section{Research Article}

Keywords: Computer Aided Diagnosis, Feature extraction, Optimized feature fusion, Classification, Machine learning, Deep CNN

Posted Date: July 1st, 2021

DOI: https://doi.org/10.21203/rs.3.rs-628691/v1

License: (c) (i) This work is licensed under a Creative Commons Attribution 4.0 International License. Read Full License 


\title{
Combination of texture and shape features using machine and deep learning algorithms for breast cancer diagnosis
}

\author{
Norhene Gargouri - Raouia Mokni • Alima Damak • Dorra Sellami • \\ Riadh Abid .
}

Received: date / Accepted: date

\begin{abstract}
Worldwide, breast cancer is a commonly occurring disease in women. Automatic diagnosis of the lesions based on mammographic images is playing an essential role to assist experts. A novel Computer-Aided Diagnosis (CADx) scheme of breast lesion classification is proposed in this paper based on an optimized combination of texture and shape features using machine and deep learning algorithms for mass classification as benign-malignant namely $\mathrm{C}(\mathrm{M}-\mathrm{ZMs})^{*}$. The main advantage of using Zernike moments for shape feature extraction is their scale, translation, and rotation invariance property, this allows omitting some of the preprocessing stages in our case. We implemented for texture
\end{abstract}

Norhene Gargouri

Digital Research center of Sfax, Technopole of Sfax, PO Box 275, Sakiet Ezzit, 3021 Sfax - Tunisia, Sfax, 3021, Sfax, Tunisie. Control and Energy Management Laboratory, National Engineering School of Sfax, Rte Soukra km 4, Sfax,Tunisia.

E-mail: norhene.gargouri@gmail.com

Corresponding author: Norhene Gargouri

Raouia Mokni

College of Computer Engineering and Science, Prince Sattam Bin Abdulaziz University, $A l-K h a r j$, Saudi Arabia.

University of Sfax, Sfax-Tunisia

E-mail: r.mokni@psau.edu.sa

Corresponding author: Raouia Mokni

Alima Damak

Control and Energy Management Laboratory, National Engineering School of Sfax, Rte Soukra km 4, Sfax, Tunisia.

Dorra Sellami

Control and Energy Management Laboratory, National Engineering School of Sfax, Rte Soukra km 4, Sfax, Tunisia.

Riadh Abid

EL Farabi Medical Imaging Center, Tunisia, Sfax. feature extraction the Monogenic-Local Binary Pattern taking the advantage of lower time and space complexity because monogenic signal analysis needs fewer convolutions and generates more compact feature vectors. Therefore, we used Zernike moments for shape feature extraction due to their scale, translation, and rotation invariance property, this allows omitting some of the preprocessing stages in our proposed system. The proposed system proves its performance on some challenging breast cancer cases where the lesions exist in dense breast tissues. Validation has been undertaken on 520 mammograms from the Digital Database for Screening Mammography Database (DDSM), yielding an accuracy rate of $99.5 \%$.

Keywords Computer Aided Diagnosis · Feature extraction - Optimized feature fusion - Classification · Machine learning · Deep CNN

\section{Introduction}

Breast cancer is the most leading type Worldwide. Breast abnormalities are a frequent finding on mammography, they are the first sign of cancer, making possible an efficient early diagnosis [1]. Accordingly, a method to decrease unnecessary breast biopsies is highly desirable essentially because a high number of patients are unnecessarily undergoing this operation with all the associated risks and costs [2]. In recent years, several studies have documented the increase in medical imaging; images today are considered not only to reflect human anatomy and physiology but also as pure data sources [3][4]. The introduction of computer-aided programs for early diagnosis has achieved a lowering of the mortality rate. 
In this study, we propose a new system based on the fusion of computer extracted features from mammographic images in combination with different classification tools. The proposed system allow to distinguish benign from malignant lesions avoiding unnecessary biopsy. It is called $\mathrm{C}(\mathrm{M}-\mathrm{ZMs}) *$ : "C" represents different classification strategies, "M" represents the Monogenic Gray Level and Local Difference (Monogenic-GLLD) algorithm, "ZMs" represents the well-known Zernike Moments algorithm, and "*" refers to optimized feature fusion. The $\mathrm{C}(\mathrm{M}-\mathrm{ZMs}) *$ model focuses on accomplishing breast mass diagnosis in mammographic images. The rest of this paper is organized as follows: Section 2 summarizes some recent works. Section 3 presents the proposed system $\mathrm{C}(\mathrm{M}-\mathrm{ZM})^{*}$. Section 4 details the experimental results. Finally, in the last section, conclusions and suggestions for future works are presented.

\section{Related work}

In this section, we present an overview of recently related works distinguishing malignant calcification's from benign ones. Recently, several studies based on deep learning in the field of breast cancer diagnosis are developed. Jadoon et al. [5] presented a novel classification algorithm based on a deep learning method. The proposed system targets normal, malignant, and benign cases. In this model, they were based on two methods, Convolutional Neural Network-Discrete Wavelet (CNNDW) and Convolutional Neural Network-Curvelet Transform (CNN-CT). Mammogram patches are used as dataset. In the enhancement stage, the authors applied the Contrast Limited Adaptive Histogram Equalization (CLAHE). Dense Scale Invariant Feature (DSIFT) for all subbands is extracted. Softmax layer and Support Vector Machine (SVM) layer are applied to train CNN for classification. The accuracy rate of the proposed approaches CNN-DW and CNN-CT are $81.83 \%$ and $83.74 \%$, respectively. The main disadvantage of CLAHE method is the tendency to over-amplify noise in homogeneous regions in the case of mammographic images, so, the proposed algorithm do not perform very well. Besides, in Soriano et al. [6] a comparison of two different schemes of mammogram classification based on CNN was developed. The main difference between these two schemes is based on the number of bits per image pixel, and the proposed feature extraction approaches. The authors used 1070 mammograms from the DDSM database. The proposed model has an accuracy of $85 \%$, in this case the validation accuracy is very low. In 2017 [7], Lotter et al. described a multi-scale CNN. Authors first train CNN-based patch classifiers on segmentation masks of lesions in mammographic images. Then they used the learned features to make the final decision. This approach achieves an Area Under Curve (AUC) of 0.92 . The proposed End-to-end (E2E) learning model requires very large amount of data in order to perform better than other techniques, this increases cost to the users. Al-masni et al. [8] in 2018 proposed a new CADx system based on four main stages: pre-processing, feature extraction with deep convolutional networks, mass detection, and classification using Fully Connected Neural Networks. In this study, they used 600 mammograms from the DDSM database and an augmented data of 2,400 with the information of the lesions and their types in the training and testing of the proposed CADx. The trained YOLO-based CADx system discriminates benign cases from malignant ones with an accuracy of $97 \%$ (AUC=0.97). yet the acceptable result obtained but the literature proves that YOLO struggles to detect close objects because each grid can propose only 2 bounding boxes. So, in the case of diagnosis malignancy it cannot be used because lesions are generally very tiny and this model struggles to detect small objects. Furthermore, error analysis of YOLO compared to Fast R-CNN shows that it makes a high number of localization errors [9].

The state-of-the-art of some related works based on classical CADx systems is presented below, Beura et al. [11] proposed a mammogram classification scheme allowing benign-malignant classification of the breast lesions. The authors used gray-level co-occurrence matrix (GLCM) and 2D-Discrete Wavelet Transform (2DDWT) as features for a Region of interest (ROI) of a mammogram. The extracted features are then used in a backpropagation neural network classifier for classification. An accuracy of $95.73 \%$ and $96.02 \%$ is obtained for the Mammographic Image Analysis Society (MIAS), and DDSM datasets, respectively. Demonstrating the effectiveness of the textons generated by these features classifying breast datasets. However, this approach does not consider deeper image features. The proposed algorithm cannot fully describe deep image features. Zhang et al. [12] proposed a new model named DE-Ada*. They extracted a set of features to characterize mammograms. Each weight is calculated to achieve mid-level feature fusion. Finally, they designed two voting-based ensemblelearning strategies to develop late feature fusion. The proposed model has an AUC of 0.97. Xie et al. [13] proposed a new system for breast cancer diagnosis based on an Extreme Learning Machine (ELM). The pre-processing stage is based on removing the pectoral muscle. Hough transform algorithm was applied for the ROI, 32 grey level, and texture features were extracted from mammographic images. The resultant accuracy was 96.02 $\%$. The major drawback of this paper is that the pro- 
posed method applied to remove pectoral muscle does not provide good result if the contour of the pectoral muscles is a curve. Table 1 summarizes the above recent studies done for benign-malignant classification.

\section{The Proposed System}

The proposed methodology is based on different stages. The first one is image acquisition (collect of benign and malignant in mammographic images from the DDSM database). The digital mammograms are then enhanced, we obtain two sets of ROIs (segmented ROIs and nonsegmented ones), which are processed for feature extraction by two different algorithms and fused. The obtained features are further applied to classify the masses as benign-malignant. Fig.1 shows a representation of the proposed methodology, the extracted ROIs are divided into two sets for training and test.

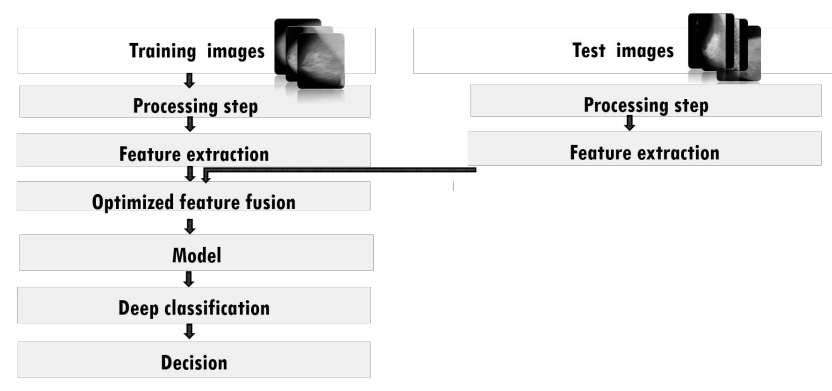

Fig. 1: General flow chart of the proposed method.

\subsection{Pre-processing}

It can be seen that the object area of a mammographic image contains the pectoral muscles, it is unwanted for the texture, shape analysis, and classication. In this work, ROIs are extracted based on the recommendations of an expert and masses are segmented. Finally, to evaluate overall performance, we compare the results with those provided by the expert. Fig. 2 illustrates the diagram of the proposed approach.

\subsubsection{Extraction of the region of interest}

In this phase, we extract the ROIs containing abnormalities based on broad expert opinion that may be useful in guiding our research (cf. Fig. 3). Fig. 4 shows some extracted ROIs containing benign and malignant lesions in the four different breast density calsses, namely ACR1, ACR2, ACR3 and ACR4. The mammographic

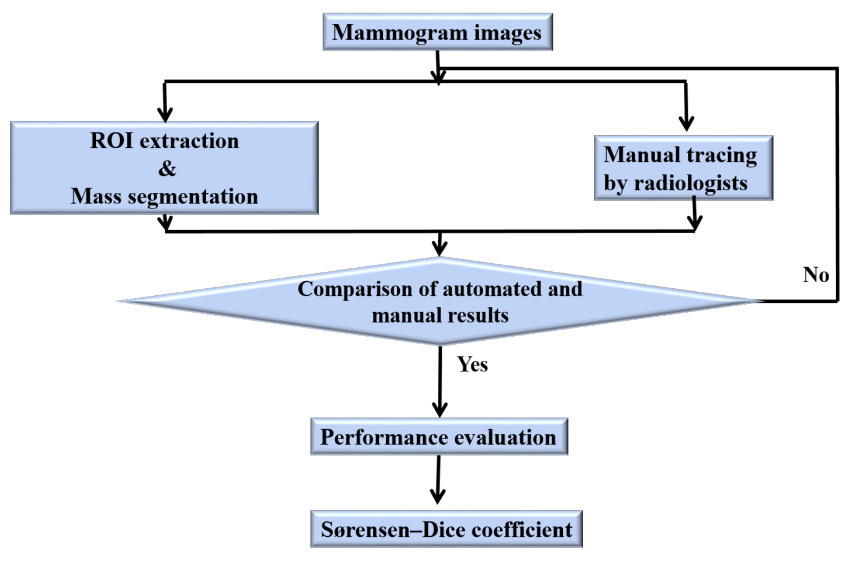

Fig. 2: Pre-processing flow diagram.
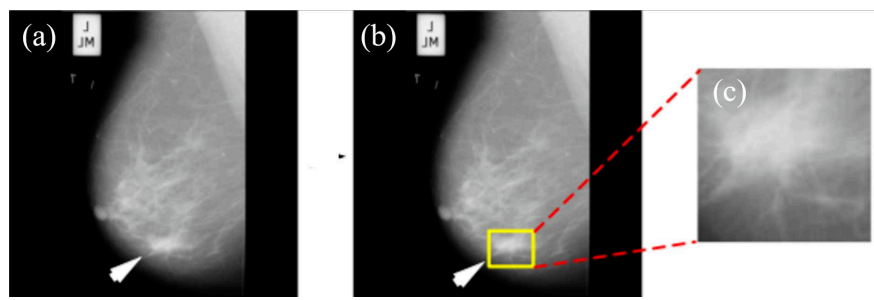

Fig. 3: ROI generation process (a) Original image (b) Manual cropping (c) ROI.

image is a correspondence between the gray levels and the density of the breast tissue. The various types of abnormalities constitute a transformation of breast tissue, resulting in visible areas in mammographic images.
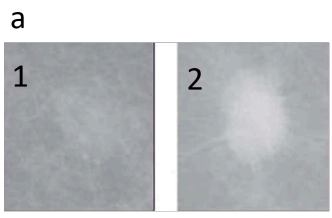

b
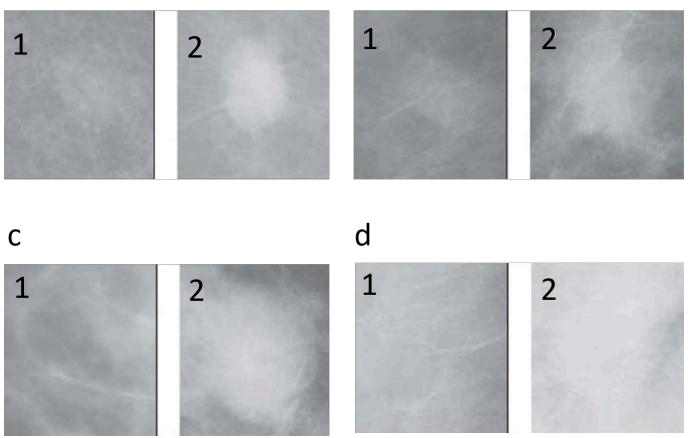

Fig. 4: Examples of ROIs with different types of tissues extracted from the DDSM database. (1) represent benign class and (2) malignant ones, (a) Almost entirely fatty tissues (b) Scattered fibro-glandular tissues (c) Hetereogeneously dense tissues (d) Extremely dense tissues. 


\begin{tabular}{|c|c|c|c|c|}
\hline Author & Year & Data Source & Techniques & Results \\
\hline Beura et al. [11] & 2015 & DDSM and MIAS & $\begin{array}{l}\text { GLCM, 2D-DWT and } \\
\text { BPNN }\end{array}$ & $\begin{array}{l}\text { Acc }(\text { MIAS })=96.02 \%, \text { AUC }(\text { MIAS })=0.9659, \\
\text { Acc }(\text { DDSM })=95.73 \%, \text { AUC }(\text { DDSM })=0.97\end{array}$ \\
\hline Xie et al. [13] & 2016 & DDSM and MIAS & ELM & $\begin{array}{l}\text { Acc }(\text { MIAS })=94.2 \%, \text { AUC }(\text { MIAS })=0.9504, \\
\text { Acc }(\text { DDSM })=97.4 \%, \text { AUC }(\text { DDSM })=0.9761\end{array}$ \\
\hline Jadoon et al. [5] & 2017 & DDSM & $\mathrm{CNN}-\mathrm{CT}$ & $\mathrm{Acc}=83.74 \%$ \\
\hline Soriano et al. [6] & 2017 & DDSM & CNN & Acc $=85 \%$ \\
\hline Lotter et al. [7] & 2017 & DDSM & Multi-Scale CNN & $\mathrm{AUC}=0.92$ \\
\hline Al-masni et al. [8] & 2018 & DDSM & CNN (YOLO) & $\mathrm{AUC}=0.96$, Acc $=97 \%$ \\
\hline Zhang et al. [12] & 2020 & DDSM & DE-Ada* & $\mathrm{AUC}=0.97$ \\
\hline
\end{tabular}

Table 1: Previously developed approaches on mammographic abnormality classification.

\subsubsection{Segmentation}

Image segmentation is crucial for medical image interpretation; there are different methods today among mammographic image lesion segmentation. The ideal lesion segmentation for mammographic images did not exist, so for mammographic images presenting lesions, there are still different possible segmentation techniques[14] [15]. Segmentation by Region Growing (RG) is well known, fast, and simple [16], but the choice of the seed point is very important, so to get a good segmentation, we are based on the expertise of a specialized medical doctor. In the RG algorithm presented in Algorithm 1, we are based on these steps:

We fix the points known as seeds $\left(p_{\text {init }}\right)$ based on expert opinion.

We fix the threshold.

Neighbors satisfying the homogeneity condition are added to the region, $i$ corresponds to the identification of the region.

Repeat the last step for each of the newly added pixels; stop if no more pixels can be added, $n$ and $m$ are the numbers of points and obtained regions respectively.

In the segmentation stage, results are compared with the manual tracings obtained by an experienced radiologist. We apply morphological operations to remove small details surrounding the lesion. As shown in Fig. 5, the details surrounded by boxes are present in the most obtained mass segmentation images. Performance evaluation is performed using the SørensenDice Similarity Coefficient (DSC).

\subsubsection{Segmentation validation metrics}

For the test and validation, validation metrics are described in the following subsections [17]:

Subjective validation:

An expert radiologist, from the FARABI Radiology Center (Sfax-Tunisia), provides the manual tracing, it val-
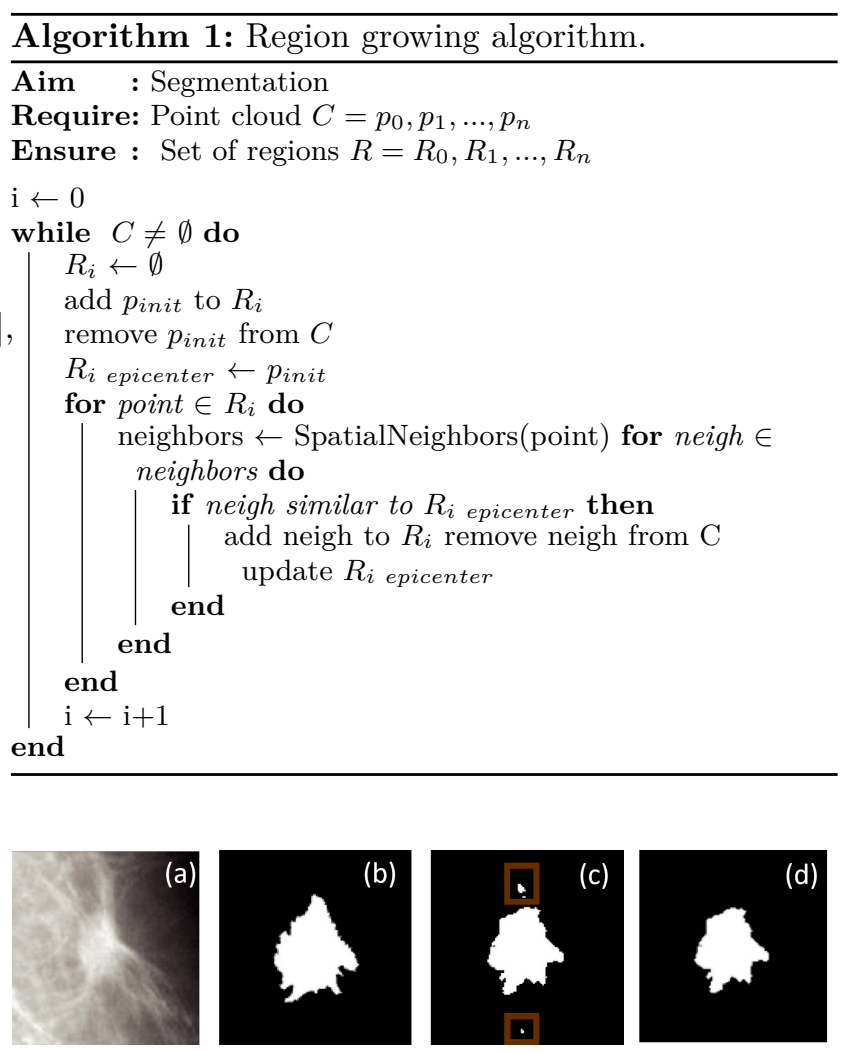

Fig. 5: Segmentation result, (a) Input mass , (b) Groundtruth mass, (c) Generated segmantation before pre-processsing and (d) Generated segmantation after pre-processsing .

idates the obtained results.

Objective validation:

The defined ground truth is extracted to detect if the crossing with segmented lesions is nonempty. We compute the DSC as segmentation accuracy [18], [19]. The DSC metric is defined by the following equation:

$D S C=\frac{2 \times \operatorname{card}(A) \cap \operatorname{card}(B)}{\operatorname{card}(A)+\operatorname{card}(B)} \times 100$ 
where $\operatorname{card}(A)$ and $\operatorname{card}(B)$ are the number of pixels in segmented lesion $\mathrm{A}$ and in the ground truth $\mathrm{B}$ respectively.

\subsection{Feature extraction}

In the proposed system, we are based on mass shape and mass texture features (cf. Fig. 6), the algorithms are detailed in the following subsections.

\subsubsection{The proposed technique Monogenic Gray Level and Local Difference-based texture feature extraction}

Texture analysis plays an important role in mammographic image analysis fields ([20] [21] [22] [23]). The proposed technique of texture Monogenic-GLLD provides a new 3-D feature vector based on joining the Gray Level and Local Difference (GLLD), the LP, and the LS information.

- The monogenic signal and the monogenic curvature tensor.

According to [24], for an image $I(\alpha)$, we can define an intrinsic dimension that expresses the number of degrees of freedom necessary to describe the local structure. The monogenic signal (MG) is an effective instrument for analyzing 1D and 2D signals in case of invariable rotation. It is based on the first order of the Riesz transform. The representation of the Riesz Kernel (RK) in 2D is as follows:

$$
\left\{\begin{array}{r}
\left(R K_{x}(\alpha), R K_{y}(\alpha)\right)=\left(\frac{x}{2 \pi|\alpha|^{3}}, \frac{y}{2 \pi|\alpha|^{3}}\right) \\
\alpha=(x, y) \in \Re^{2}
\end{array}\right.
$$

The Fourier transform of the RK kernel is given by:

$$
\left\{\begin{array}{r}
\left(T F_{u}(\beta), T F_{v}(\beta)\right)=\left(-i \frac{u}{|\beta|},-i \frac{v}{|\beta|}\right) \\
\beta=(u, v) \in \Re^{2}
\end{array}\right.
$$

The MG is presented as a fusion of the image and its Riesz transform:

$$
\begin{aligned}
I(\alpha)_{M}= & \left(I(\alpha), R K_{x}\{I\}(\alpha), R K_{y}\{I\}(\alpha)\right) \\
= & \left(I, R K_{x} * I, R K_{y} * I\right)
\end{aligned}
$$

Where $*$ corresponds to the convolution operator. In [24] and [25], the local orientation is computed as follows:

$$
\theta=\arctan \frac{R K_{y}\{I\}}{R K_{x}\{I\}}, \theta \in[0, \pi)
$$

Moreover, for any intrinsic 1D signal $I(x)$, we can demonstrate that:

$$
\begin{gathered}
\sqrt{R K_{x}^{2}\{I\}(0,0), R K_{y}^{2}\{I\}(0,0)}=\left|\left(h_{1} * I \theta\right)(0)\right| \\
=\left|-\frac{1}{\pi} \int_{t \in \Re} \frac{I(t \cos \theta, t \sin \theta)}{t} d t\right|
\end{gathered}
$$

$h_{1}(t)=\frac{1}{\pi t}$ and $h_{1} * I$ corresponds to the the Hilbert measure of $I$ respectively. The LP of the $1 \mathrm{D}$ signal $I(\alpha)$ is defined as follows:

$\varphi=\operatorname{atan} 2\left(\sqrt{R K_{x}^{2}\{I\}+R K_{y}^{2}\{I\}, I}\right), \varphi \in[0, \pi)$

Thus, the LP is calculated invariantly by rotation. The monogenic curvature tensor is based on higher orders of the Riesz transform [24] and is expressed as follows:

$C T_{e}=\left[\begin{array}{l}R K_{x}\left\{R K_{x}\{I\}\right\}, R K_{x}\left\{R K_{y}\{I\}\right\} \\ R K_{x}\left\{R K_{y}\{I\}\right\}, R K_{y}\left\{R K_{y}\{I\}\right\}\end{array}\right]$

We can see that $C T_{e}$ is composed by the second order of the transform of the Riesz transform RK, its determinant is defined by:

$$
\begin{aligned}
& \text { determinant }\left(C T_{e}\right)=\operatorname{det}\left(C T_{e}\right) \\
= & \left(R K_{x}\left\{R K_{x}\{I\}\right\}\right)\left(R K_{y}\left\{R K_{y}\{I\}\right\}\right) \\
& -\left(R K_{x}\left\{R K_{y}\{I\}\right\}\right)^{2}
\end{aligned}
$$

The sign of the determinant of $C T_{e}$ is a rotation invariant measure. It is related to the shape of the curvature of the surface. If the curvature is elliptical, the sign is positive, otherwise, when the curve is hyperbolic, the sign is negative.

Lin Zhang et al. [27] investigated the problem of rotation invariant texture classification and proposed the Monogenic Local Binary Pattern, this latter is a new textural representation based on the combination of the local phase information, the LS type and the $L B P^{r i u 2}[26]$. The main limitation of using the LBP code is that it may give the same results with two completely different gray levels when the differences with the neighbors are the same. In [20], we improved the LBP algorithm to allow the extraction of more relevant texture feature attributes from mammographic images to reduce the false positive and true negative rates based on the gray level and local difference information. In the following, we present details of the GLLD algorithm.

- Gray Level and Local Difference

In the GLLD algorithm [20], we computed the average for each $[3 \times 3]$ neighborhood noted $g_{\text {cmean }}$, then we attributed this value to the central pixel. The 


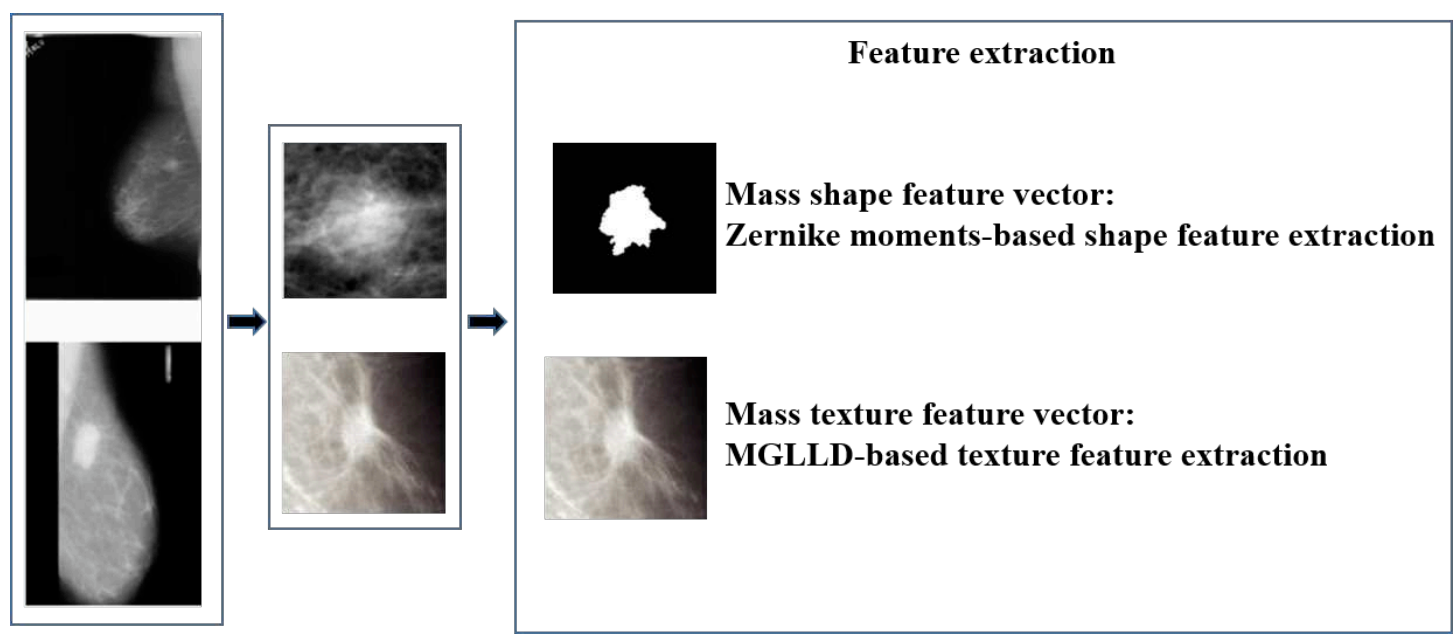

Fig. 6: Feature extraction stage.

main idea of the proposed algorithm is presented as follows:

$T=t\left(g_{\text {cmean }}, g_{0}-g_{\text {cmean }}, \ldots, g_{P-1}-g_{\text {cmean }}\right)$

where $g_{\text {cmean }}$ and $P$ corresponds to the proposed value of the central pixel and its neighbors placed on a circle of radius $R$. The difference between $g_{\text {cmean }}$ and $g_{p}$ is expressed as follows:

$\operatorname{dif} f_{p}=g_{p}-g_{\text {cmean }}$

Where $\operatorname{dif} f_{p}=\left[\operatorname{dif} f_{0}, \ldots\right.$, dif $\left.f_{P-1}\right]$ and the resulted vector is decomposed as follows:

$\operatorname{dif} f_{p}=m_{p} . s_{p}$

where $s_{p}$ and $m_{p}$ corresponds to the sign of the differences and the absolute value respectively and they are computed as follows:

$s_{p}=\left\{\begin{array}{r}\text { 1ifdiff } f_{p}>=0 \\ -1 e l s e\end{array}\right.$

and

$m_{p}=\left|\operatorname{diff} f_{p}\right|$

The result is two vectors, the sign and the modulus vector $\left(\left[s_{0}, \ldots, s_{P-1}\right]\right.$ and $\left[m_{0}, \ldots, m_{P-1}\right]$ respectively). The sign coding component named $S G L L D$, is defined as:

$$
\begin{aligned}
& S G L L D_{P, R}=\sum_{p=0}^{P-1} s\left(g_{p}-g_{\text {cmean }}\right) 2^{p} \\
& s(x)=\left\{\begin{array}{c}
1 \text { if } x>=0 \\
-1 \text { else }
\end{array}\right.
\end{aligned}
$$

On the other hand, the (MGLLD) and is defined as follows:

$M G L L D_{P, R}=\sum_{p=0}^{P-1} t\left(m_{p}, c\right) 2^{p}$

where $t(x, c)$ is defined by:

$t(x, c)=\left\{\begin{array}{r}1, x>=c \\ 0, x<c\end{array}\right.$

$c$ corresponds to the mean value of the whole image. The value of the central pixel is also coded as:

$C G L L D_{P, R}=t\left(g_{\text {cmean }}, c\right)$

In our previous work [20], we proposed to concatenate the three obtained vectors $(S G L L D),(M G L L D)$ and $(C G L L D)$, the output vector is the named GLLD vector, Fig. 7 illustrates the principle of the GLLD algorithm. The $G L L D_{P, R}^{r i u 2}$ is considered an efficient

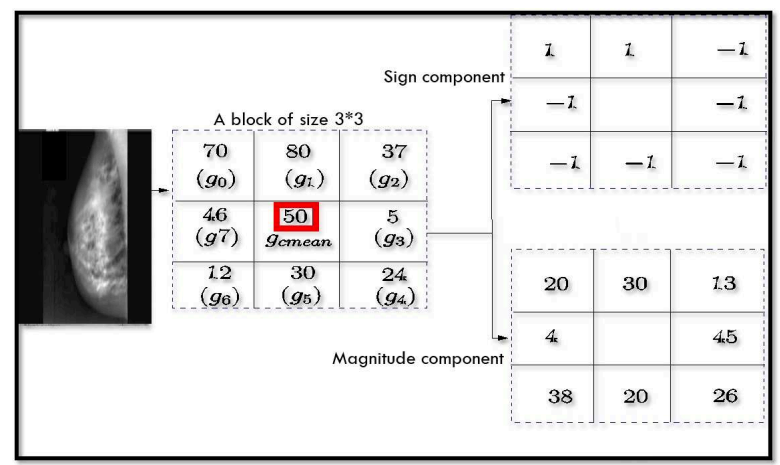

Fig. 7: An example of GLLD algorithm.

tool in mammographic images texture classication 
[20], it provides high robustness against the rotation of a texture, but it can code different textures (having undergone non-uniform rotations giving rise to phase differences in the image) in an identical manner. Benign-malignant mass classication accuracy requires special attention to take into account Local Phase (LP) shifts and Local Surface (LS) information in the texture image. In this context, it is relevant to combine the GLLD operator with the monogenic caracteristics to overcome this GLLD limitation.

- Monogenic Gray Level and Local Difference

The LP code and the LS are described in equations 20 and 21 respectively as:

$$
\begin{aligned}
\varphi_{c} & =\lceil\varphi /(\pi / M)\rceil \\
\zeta_{c} & =\left\{\begin{array}{cc}
0 i f & \operatorname{det}\left(C T_{e}\right) \leq 0 \\
1 & \text { else }
\end{array}\right.
\end{aligned}
$$

where $\varphi_{c}$ is the local phase. In the experimental part, we set $\mathrm{M}$ to 5 and $\varphi_{c}$ is defined within $[1,5]$.

We don't use an exact value fo $\operatorname{det}\left(C T_{e}\right.$ defined in equation (21), but its binarized value. So, a new 3$\mathrm{D}$ feature vector is developed by fusing $\varphi_{c}, \zeta_{c}$ and $G L L D_{P, R}^{r i u 2}$, namely $M G L L D_{P, R}^{r i u 2}$.

\subsubsection{Zernike moment-based shape feature extraction}

Zernike moments are invariant to rotations and translations. To make them invariant to pixel gray level, they are normalized and resized. To overcome the problem of translation sensitivity of these descriptors, we have used different outcomes. We started with a binarization of the image. Then, we proceeded to the filling of the shape. This leads to a translation of the centroïd of the mass towards the center of the image. We then computed the coordinates of the centroïd (co,ro) as follows:

$c o=\left[\frac{\sum_{c=0}^{N-1} \sum_{c=0}^{N-1} c f(c, r)}{\sum_{c=0}^{N-1} \sum_{c=0}^{N-1} f(c, r)}\right]$

$r o=\left[\frac{\sum_{c=0}^{N-1} \sum_{c=0}^{N-1} r f(c, r)}{\sum_{c=0}^{N-1} \sum_{c=0}^{N-1} f(c, r)}\right]$

the pair $(c, r)$ determines the coordinates of the image and $f(c, r)$ represents the value of the gray level in the considered image. In the feature extraction step, for Zernike moments, in polar coordinates $(\rho, \theta)$, radial polynomials $R_{m n}(\rho)$ are defined by:

$$
R_{m}^{n}(\rho)=\sum_{s=0}^{\frac{n-|m|}{2}} \frac{(-1)^{s}(n-s) !}{(s) !\left(\frac{n+|m|}{2}-s\right) !\left(\frac{n-|m|}{2}-s\right) !} \rho^{n-2 s}
$$

Where $n$ and $m$ are integers knowing that $n \geq m, \theta$ is the azimuthal angle and $\rho$ radial distance. For an order $n$ and a repetition $m$, Zernike polynomials are widely used as basis functions of image moments and defined as :

$R_{m}^{n}(\rho, \theta)=R_{m}^{n}(\rho) \exp (j m \theta)$

where $(\rho, \theta)$ are defined on the unit disc as $j^{2}=-1$ and $R_{m n}(\rho)$ corresponds to the orthogonal radial polynomial.

Zernike moments are defined on the unit disc $\left(x^{2}+\right.$ $\left.y^{2}\right)<=1$, they are expressed as:

$Z_{\prime m}^{n}=\frac{n+1}{\pi} \iint_{\text {unit disc }} V_{m}^{n *}(\rho, \theta) f(\rho, \theta)$

where $V_{m}^{n *}$ is the complex conjugate of $V_{m}^{n}, f(\rho, \theta)$ is associated with the original image, $m=0,1, \ldots, \infty$ represents the order and $n$ is an integer such that:

$|n| \leq m$

Thus, to calculate the Zernike moments of an image $f(x, y)$, different operations must be performed. First, the region of interest is plotted using the polar coordinates on the unit disc. The pixels, located outside the disc, are not taken into account in the calculation. To go from a cartesian system to a polar system, $x$ and $y$ are defined by:

$x=\rho \cos \theta \quad y=\rho \sin \theta$

knowing that:

$\rho=\sqrt{x^{2}+y^{2}} \quad \theta=\left(\frac{y}{x}\right)$

and $\tan ^{-1}(A)$ is defined within $\frac{-\pi}{2} \leq \theta \leq \frac{+\pi}{2}$. The order $(0,0)$ of the geometric moment $m_{00}$ of the image is calculated for the step of the normalisation of the Zernike moments.

$m_{00}=\iint f(x, y) d x d y$

Then, Zernike moments of the different orders are computed as follows:

$Z_{m}^{\prime n}=\frac{n+1}{\pi} \iint_{\text {unit disc }}{ }^{*} V_{n m}^{*}(x, y) f(x, y) d x d y$

Finally, the normalisation of these descriptors is computed using $Z_{m}^{\prime n}$ knowing that:

$Z_{m}^{n}=\frac{Z_{m}^{\prime n}}{m_{00}}$

Once we get the Zernike moments $Z_{m}^{\prime n}$ in their complex form, we apply the modulus to the obtained results. 
Usually, Zernike moments with low order on the unit disc illustrate the overall shape, however high orders of these moments illustrate details of a shape to be characterized. In addition, high orders are not generally characterized by computational complexity only, but also by sensitivity to noise. In addition, they have the effect of minimizing the performance of the system if they are not precisely selected. To study their performance, we have adopted a high-order set of these moments, on the one hand, and a low-order set on the other hand, which must be extracted from the preprocessed ROIs.

For all ROIs, based on the results obtained in [28], we used the Zernike moments of order $6 . M_{z}(z=1, \ldots, 7)$, the obtained vector is named $V_{Z M s}$.

\subsection{Optimized Fusion}

Information fusion is a successful tool in medical image processing applications [29]. The fusion of multiple features will improve the system's performance. In this paper, we focus on the feature level fusion that is accomplished by concatenation of the obtained feature vectors. The proposed system is based on the fusion of $V_{Z M s}$ and $M G L L D_{P, R}^{\text {riu2 }}$ where $V_{Z M s}$ feature size is 4 and $M G L L D_{P, R}^{r i u 2}$ feature size is 540 . The obtained vector is named $V_{M G L L D-Z M s}$, with a size equal to 544 . To improve the classification accuracy and remove redundant features, we propose the algorithm based Weighted Features Fusion. In fact, only some elements of the obtained features should be selected and weighted depending on the inter-class and intra-class distances. $w_{k}$ is a feature weighting vector where $(k=1 ; 2 ; \ldots ; m)$, it is defined as follows:

$w_{k}=\left\{\begin{array}{r}0, \\ {[1, F]}\end{array}\right.$

$w_{k}$ is assigned to 0 if the feature will not be considered. $F$ is fixed to 10 . Consider a matrix of feature $M$ of size $m * n, \mathrm{~m}$ corresponds to the size of features and $\mathrm{n}$ the number of images. Thus, each $M_{k, l}$ denotes the $k^{t h}$ feature of the $l^{t h}$ element. The weights application for the $l^{\text {th }}$ element is represented as:

$B_{l}=W \odot A_{l}$

$\odot$ corresponds to the pointwise operator. $W$ is the weighting vector feature and $A$ is a feature vector. The distance is also presented as:

$$
\begin{array}{r}
\operatorname{dist}\left(B_{i}, B_{j}\right)=\sum_{k=1}^{m}\left|b_{k, i}-b_{k, j}\right| \\
=\sum_{k=1}^{m} w_{k}\left|a_{k, i}-a_{k, j}\right|
\end{array}
$$

so,

$\operatorname{dist}\left(B_{i}, B_{j}\right)=w^{T}\left|A_{i}-A_{j}\right|$

The inter-class and intra-class distances may be formulated in the same manner as follows:

$$
\begin{aligned}
\text { dist }_{\text {intra/inter }} & =\sum_{i=1}^{n} \sum_{j=i+1}^{n} \operatorname{dist}\left(B_{i}, B_{j}\right) \\
& =\sum_{i=1}^{n} \sum_{j=i+1}^{n} w^{T}\left|A_{i}-A_{j}\right| \\
& =w^{T} \sum_{i=1}^{n} \sum_{j=i+1}^{n}\left|A_{i}-A_{j}\right|
\end{aligned}
$$

The optimal-subset weighted features is obtained by maximizing and minimizing the inter-class and intraclass distances respectively while varying the weight $W$. In the proposed algorithm, we initialize the $W$ randomly within $[1, \mathrm{~F}]$.

Let's consider $W=\left[w_{1}, w_{2}, \ldots, w_{m}\right]^{T}$ the obtained vector and $k \in[1, m]$, so the $k^{t h}$ element of the repaired vector is calculated as follows:

$w_{k}^{\prime}=\left\{\begin{array}{r}F, \text { if } w_{k}>F \\ 0, \text { if } w_{k}<1 \\ w_{k}, \text { otherwise }\end{array}\right.$

After repair the newly obtained weighting vectors, we compute the Objective Values (OVs). $O_{k}^{\min }$ and $O_{k}^{\max }$ are the minimum and maximum OVs respectively of all solutions. The degree by which the $z^{\text {th }}$ solution satisfies the $k^{\text {th }}$ Objective Function (OF) is represented as follows:

$\nu_{k}^{z}=\left\{\begin{array}{r}1, \text { if } O_{k}^{z}<O_{k}^{\text {min }} \\ \frac{O_{k}^{\text {max }}-O_{k}^{z}}{O_{k}^{\text {max }}-O_{k}^{\text {min }},} O_{k}^{\text {min }}<O_{k}^{z}<O_{k}^{\text {max }} \\ 0, O_{k}^{z}>O_{k}^{\max }\end{array}\right.$

For the optimization of the OF, knowing that $z \in[1, N]$, $\nu^{z}$ is expressed as:

$\nu^{z}=\frac{\left[\nu^{z}\right]^{T}}{\sum_{l=1}^{N}\left[\nu^{l}\right]^{T}}$

For each $N$, there is a feature weighting vector $W^{z}$, the optimal vector is computes as:

$W^{o p t}=\left(W^{z}\right)^{o p t}$

where

$z^{o p t}=\max _{z} \nu^{z}$ 
So, a new subset of features is developed after the application of the weights on them. Figure presents the organigram of the proposed algorithm of optimized fusion.

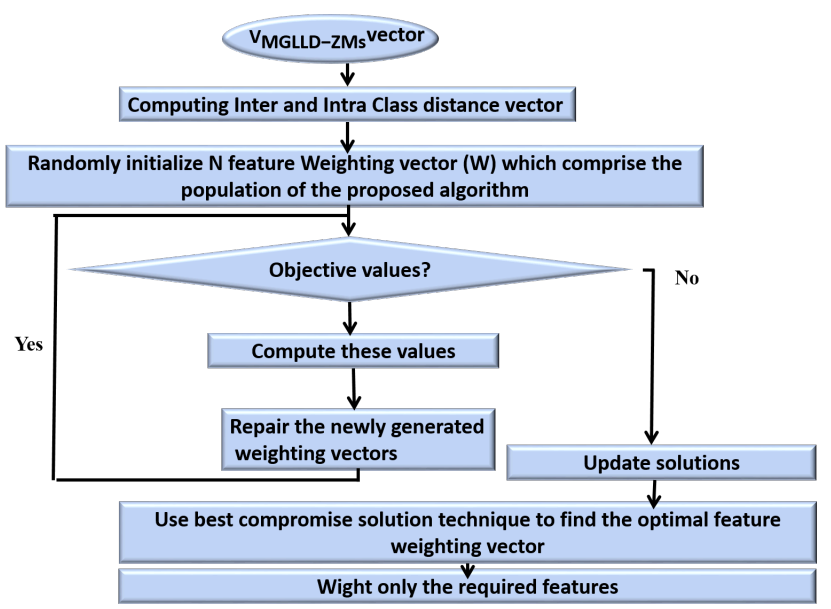

Fig. 8: Organigram of the used algorithm for feature optimization.

\subsection{Classification}

The last step in the proposed system is mass classification. To seek generality and to prove the choice of the classifier, a test of four classifiers will be undertaken. The applied classifiers (SVM, K-Nearest Neighbors (kNN), Artificial Neural Network(ANN), and Deep $\mathrm{CNN}$ ) are detailed in the following section.

\subsubsection{Support Vector Machine}

Vapnick et al. [30] proposed the SVM classifier, which is widely applied in several fields such as medical imaging and pattern recognition. It allows the discrimination between positive from negative examples, by finding a hyperplane that separates two classes. The SVM can identify the hyperplane, this latter maximizes the separation between the two classes. A kernel function $\varphi$ is utilized by SVM in the following equation:

$S(y)=\sum_{j} a_{j} \varphi\left(s_{j}, y\right)+c$

where $s_{j}, a_{j}$ and $c$ are the support vectors, the weights, and the bias respectively. $\varphi$ corresponds to a dot product in the case of a linear kernel. So, based on the sign of $S, y$ is classified as belonging to each output class.

\subsubsection{K-Nearest Neighbors}

KNN algorithm is a supervised machine learning algorithm. This classifier finds the closest data points based on Euclidean distance; Then, the voting approach is used for classification [31]. The output vector corresponds to the ratio of the elements of each class to the total number of neighbors in the used database.

\subsubsection{Artificial Neural Network}

ANNs algorithms are applied in several fields such as medical imaging and pattern recognition [32]. They draw much of their inspiration from the biological neural systems. This approach is based on adjusting weights to the best-fitting functional approximations. There are two Artificial Neural Network (ANN) approaches, specifically, Multilayer Perceptron (MLP) and Radial Basis Function (RBF) networks. This paper presents a comparison between the results obtained by MLP and RBF. for the input vector $a=(a 1, a 2, \ldots, a d)^{T}$ and $X(a)$ the activation function, the ANN output $z$ is presented as follows:

$z=X\left(\omega^{T} a_{b}\right)=X\left(\sum_{i=1}^{d} \omega_{i} a_{i}-b\right)$

where $\omega=(\omega 1, \omega 2, \ldots, \omega d)^{T}$ corresponds to the weight vector. The obtained features named $V_{M G L L D-Z M s}$ are used as input of the ANN and there is one output neuron. In the proposed architecture, one hidden layer is sufficient, with 20 hidden neurons. The RMS error approaches zero within 2000 iterations and we get a maximum mean square error of 0.001 .

\subsubsection{Deep CNN classifier}

Lately, deep learning delivered important success in several fields such as pattern recognition ([34], [35]), medical imaging [36], and biometrics [37]. Shape information extracted from deep networks is very limited [10]. In this study, we have investigated a new descriptor to analyze the texture (Monogenic-GLLD), this latter gives information about the gray level and local difference applied together, for sake of more accuracy. We used also a commonly used descriptor such as Zernike moments to analyze the shape of the segmented lesions. Thus, we get a feature vector, ready to be used as input to the deep CNN classifier. To achieve the ultimate goal, we applied a new deep classifier architecture that is a sequential one-input-one-output feed-forward. The class of malignant cases is assigned as class 1 and of benign cases is assigned as class 0 . 


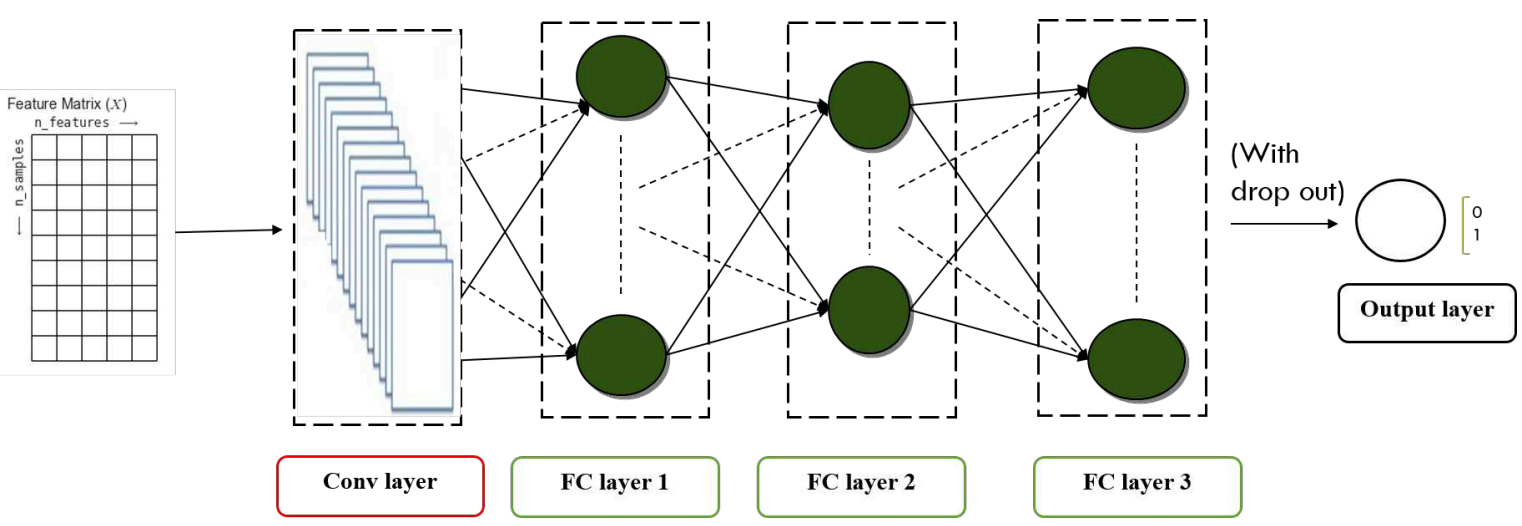

Fig. 9: Deep architecture.

As illustrated in Fig. 9, our proposed architecture is composed of one convolutional layer, three fully connected Dense layers, one Dropout layer, and one Output layer. The first layer, a convolutional one, creates 2-D convolutions with 16 kernels of size [3×3] and 'same' padding. The three fully connected hidden layers include the associated training parameters, such as the number of neurons and the activation function. These three fully connected hidden layers consist of 32 neurons each. In each fully connected layer, the nonlinear transformation is carried out by Rectified Linear Units (ReLU) as the activation function. Only the last fully connected layer contains the dropout probability with $20 \%$ probability that is performed to reduce overfitting. In the last layer, the Classification layer, two neurons are used as the number of output classes that will be predicted 0 or 1 applying the Softmax Activation Function. In the training part, we take the logarithmic loss function (binary-cross-entropy) used for the binary classification problem. We adopt several experiments in our training model with two optimizer functions such as Adam and Stochastic Gradient Descent with momentum (SGDM) and different epoch numbers such as 20, 50,100 . A study of the effect of the used optimizer functions and epoch numbers variation on the performance of our model will be illustrated below in the experimental results section. The best results have been provided by the SGDM optimization algorithm with a momentum value of 0.9 and L2regularization $\mathrm{l}=1.0000 \mathrm{e}-04$, Gradient threshold method L2norm, and by a number of epochs equal to 50. The details of these used training parameters in our Deep CNN architecture are depicted in Table 2.
Table 2: Training options of the used SGDM optimizer in our Deep CNN architecture.

\begin{tabular}{|l|l|}
\hline Parameter & Value \\
\hline Number of iterations & 450 \\
\hline Epochs Number & 50 \\
\hline Optimizer & SGDM \\
\hline Gradient threshold method & L2norm \\
\hline Momentum & 0.9 \\
\hline L2regularization & $1.0000 \mathrm{e}-04$ \\
\hline
\end{tabular}

\section{Experimental validation}

\subsection{Experimental dataset}

The proposed approach is tested based on the publicly available DDSM database containing 2620 patients (see Table 3). For each patient, there are the Cranio Caudal and Medio Lateral Oblique views [33]. The DDSM database contains delineations of biopsy-proven abnormalities, the Metadata and Breast Imaging Reporting And Data System (BI-RADS) assessment code for each abnormality. The provided delineations in the DDSM database are not adequate for segmentation results validation, so, we were based on the extraction of ROIs on manual segmentation entertained by an expert radiologist.

Table 3: DDSM Database.

\begin{tabular}{|l|l|}
\hline Number of mammgorams & 10.239 \\
\hline Image file format & DICOM \\
\hline Size of images (in pixels) & $1024^{*} 1024$ \\
\hline Lesion type & All kind \\
\hline Views & MLO, CC \\
\hline Public access & Available \\
\hline
\end{tabular}




\subsection{Performance metrics}

TP, FP, TN, and FN correspond to the true positive, false positive, true negative rate, and false negative cases respectively. ROC curve is a plot of the false positive rate versus the true positive rate as shown in Fig. 10. The AUC (Area Under the Curve) is the measure of the ability of a classifier to distinguish between different classes, it is a universal metric that may be applied to compare diverse tests. The value of AUC satisfies this condition:

$0 \leq A U C \leq 1$

The system is more accurate when both distributions are disjoint, yielding an AUC value which is closer to one. The accuracy, sensitivity, and specificity are also

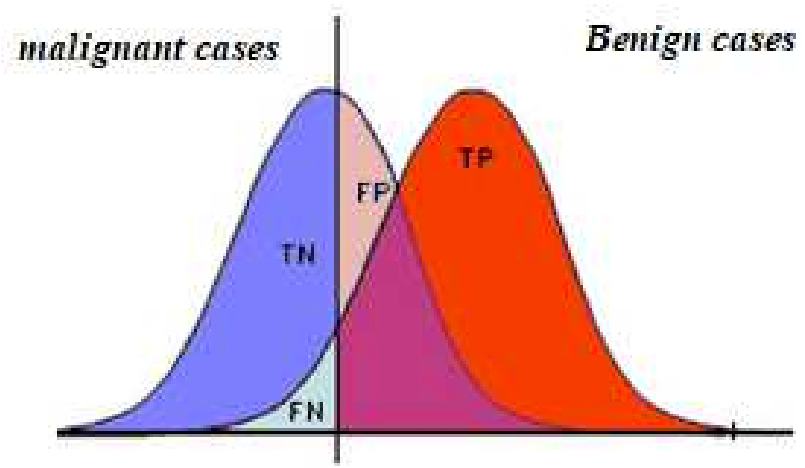

Fig. 10: Distribution of the classes on the classifier output.

so important to decide about the performance of the proposed system. They are defined as follows:

Accuracy $=\frac{T P+T N}{T P+T N+F P+F N}(\%)$

A system with high accuracy has high performance.

Sensitivity $=\frac{T P}{T P+F N}(\%)$

$1-$ Specificity $=1-\frac{T N}{T N+F P}(\%)$

\subsection{Evaluation of the proposed approach}

To test the performance of the proposed system, we used 1040 ROIs where 520 shape ROIs and 520 texture ROIs were extracted from the DDSM database (cf. Fig. 11). For each one, we split into $70 \%$ training and $30 \%$ test as follows:
- 120 samples for tests: 60 benign masses and 60 malignant masses.

- 400 samples for training: 200 benign masses and 200 malignant masses.

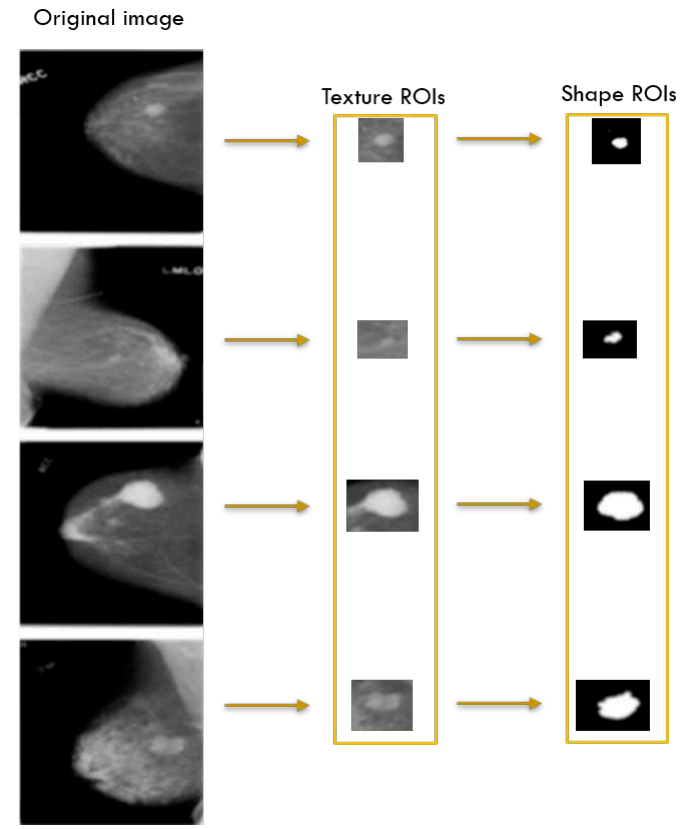

Fig. 11: Samples of extracted ROIs.

In the segmentation stage, the obtained results for the DSC coefficient reach a rate of $90 \%$. This rate demonstrates the performance of the applied algorithm in the segmentation of suspicious tissue in mammographic images. The obtained results of segmentation are validated objectively by a radiologist. Fig. 12 illustrates examples of ROI segmentation for malignant and benign masses.

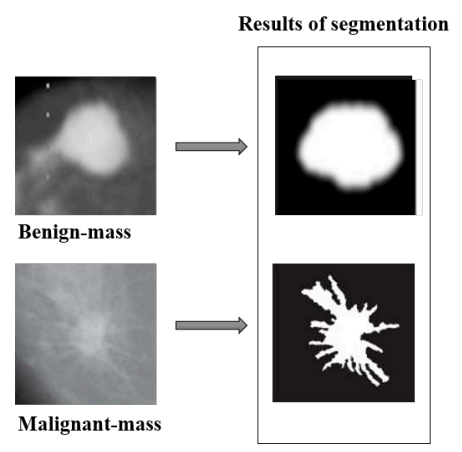

Fig. 12: Example of ROI segmentation result. 
From the results presented in Table 4, we can conclude that the results of the fusion of the two descriptors when applying the Deep CNN classifier provide the best results $(\mathrm{AUC}=0.98)$. Moreover, to prove the choice of

Table 4: Comparison of the results of the different features.

\begin{tabular}{|l|l|}
\hline Classifier & AUC \\
\hline Feature fusion & $\mathbf{0 . 9 8}$ \\
\hline Texture feature & 0.97 \\
\hline Shape feature & 0.96 \\
\hline
\end{tabular}

the selected function MLP for ANN classifier, we provide in Table 5 a comparison between MLP and RBF function illustrated in this table. MLP achieves best result $(95 \%)$.

Table 5: Comparison between MLP and RBF

\begin{tabular}{|l|l|l|}
\hline Number of epochs & MLP & RBF \\
\hline Accuracy (\%) & $\mathbf{9 7}$ & 95 \\
\hline
\end{tabular}

Table 6: Comparison of the different methods of classification.

\begin{tabular}{|l|l|l|l|l|}
\hline \multirow{2}{*}{ Classifier } & \multicolumn{3}{|l|}{ Metrics (\%) } \\
\cline { 2 - 5 } & AUC & ACC & Sensitivity & Specificity \\
\hline Deep CNN & $\mathbf{0 . 9 8}$ & $\mathbf{9 9 . 5}$ & 98.61 & 96.73 \\
\hline MLP & 0.96 & 97 & 96.02 & 94.32 \\
\hline KNN & 0.93 & 90.94 & 92.52 & 89.07 \\
\hline SVM & 0.92 & 89.77 & 92.06 & 87.77 \\
\hline
\end{tabular}

Furthermore, a comparative study presented in Table 6 and Fig. 13 proves that the Deep CNN classifier provides the best accuracy compared to other classifiers. To validate the choice of epoch number and optimizer function, Table 7 reveals the effect of varying the number of the epoch, and Table 8 proves the choice of the optimizer.

Table 7: Variation of the number of epochs.

\begin{tabular}{|l|l|l|l|}
\hline Number of epochs & 20 & 50 & 100 \\
\hline Accuracy (\%) & 98.8 & $\mathbf{9 9 . 5}$ & 98 \\
\hline
\end{tabular}

Fig. 14 aims to ensure that no overfitting is happening and that the Deep CNN classifier model performs well on the training data as well as on validation data. This figure represents an accuracy graph for a better

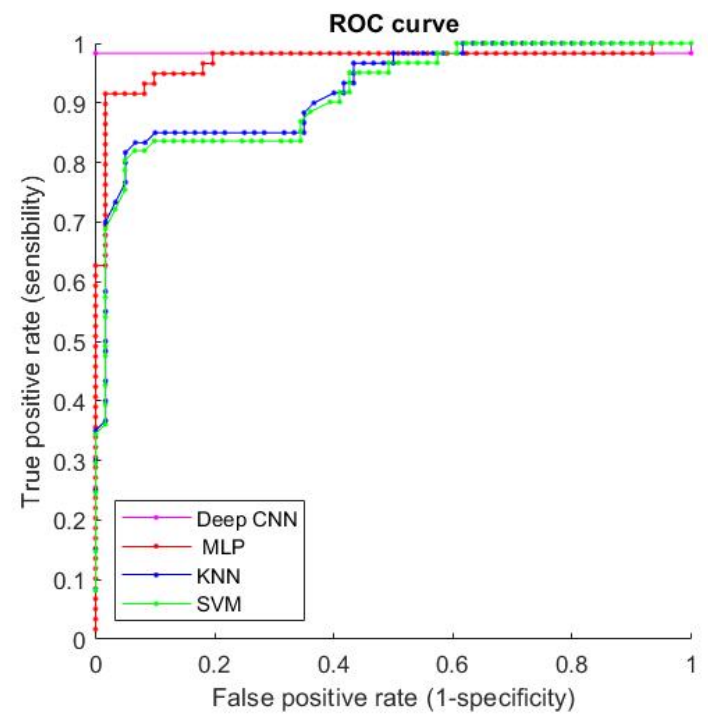

Fig. 13: ROC curve.

Table 8: Effect of the used optimized function variation.

\begin{tabular}{|l|l|l|}
\hline Used optimized function & adam & sgdm \\
\hline Accuracy (\%) & 92 & $\mathbf{9 9 . 5}$ \\
\hline
\end{tabular}

understanding of our proposed model and results. In fact, from the top of this figure, the iteration is plotted on the x-axis, and accuracy is plotted on the y-axis. In the same way, a loss can also be determined from the bottom of this figure, it shows plotted values of model loss and illustrates the plot of the model loss over the epochs or iterations for the training (loss) and validation sets. According to this figure, iteration is plotted on the $\mathrm{x}$-axis, and Loss is plotted on the y-axis. The Deep CNN classifier model shows comparable performances on the training and validation data, as shown in Fig. 14. In addition, a comparative study was provided, Table 9 , presents the results of comparison based on different $A_{z}$ values of our proposed system with some related work presented in section 2 . The comparison proves the efficiency of the proposed system for benign-malignant mass classification. For instance, [7], [8], [13], [11] and [12] which used the same database obtained $A_{z}$ values $0.92,0.96,0.97,0.97$ and 0.97 respectively, while we obtained an $\mathrm{AUC}=0.98$.

\section{Conclusion}

In this paper, we propose a novel system $\mathrm{C}(\mathrm{M}-\mathrm{ZMs})^{*}$ for mass classification based on mammographic modality. In the pre-processing stage, the regions containing masses are segmented by RG and morphological operators. Then, the new optimized feature vector based 


\begin{tabular}{l|l|}
\hline \\
Fig.
\end{tabular}

on shape and texture is built, and finally classification based on machine learning and Deep CNN classifier is developed to ensure a fair comparison. The experimental results demonstrate that the CADx system has the best accuracy in the recognition of benign and malignant breast tumors with respect to the related work. In this paper, we proposed a new CAD allowing to diagnose breast lesions. We hope that the proposed system will assist the experts to make more accurate and quick decisions. Future work includes extending the proposed algorithm for Breast Imaging-Reporting And Data System (BIRADS) classification and to apply the used techniques to others pathology fields.

\section{References}

1. C. Allemani, T. Matsuda, V. Di Carlo, R. Harewood, M. Matz, M. Niksic, et al., Global surveillance of trends in can- cer survival 200014 (CONCORD-3): analysis of individual records for 37513025 patients diagnosed with one of 18 cancers from 322 population-based registries in 71 countries, Lancet 391 (10125), 10231075, 2018.

2. M. Rominger, C. Wisgickl, N. Timmesfeld, Breast microcalcifications as type descriptors to stratify risk of malignancy: a systematic review and meta-analysis of 10665 cases with special focus on round/punctate microcalcifications, RofoFortschr. Auf Dem Gebiet Der Rontgenstrahlen Und Der Bildgebenden Verfahren. 184 (12), 11441152, 2012.

3. P. Lambin, E. Rios-Velazquez, R. Leijenaar, S. Carvalho, R.G.P.M. van Stiphout, P. Granton, et al., Radiomics: extracting more information from medical images using advanced feature analysis, Eur. J. Cancer 48 (March (4)), 441446, 2012.

4. R.J. Gillies, P.E. Kinahan, Hricak H. Radiomics, Images are more than pictures, they are data, Radiology 278 (2), 563577,2016 .

5. M. Jadoon and Q. Zhang and I. Haq and Sharjeel Butt and Adeel Jadoon, Three-Class Mammogram Classification Based on Descriptive CNN Features, BioMed Research International, 2017.

6. M. Soriano, D; Aguilar, C; Ramirez-Morales, I; Tusa, E; Rivas, W; Pinta, Mammogram classification schemes by using convolutional neural networks, Int. Conf. Technol. Trends, pp. $7185,2017$.

7. W. Lotter, G. Sorensen, D. Cox, A Multi-Scale CNN and Curriculum Learning Strategy for Mammogram Classification. In: MICCAI Workshop DLMIA (2017).

8. Al-masni, M. A., Al-antari, M. A., Park, J.-M., Gi, G., Kim, T.-Y., Rivera, P., Kim, T.-S., Simultaneous detection and classification of breast masses in digital mammograms via a deep learning YOLO-based CADx system, Computer Methods and Programs in Biomedicine, 157, 8594, 2018.

9. Du, Juan. "Understanding of object detection based on CNN family and YOLO." Journal of Physics: Conference Series. Vol. 1004. No. 1. IOP Publishing, 2018.

10. Baker N, Lu H, Erlikhman G, Kellman PJ, Deep convolutional networks do not classify based on global object shape. PLOS Computational Biology 14(12), 2018. 
11. S. Beura, B. Majhi, S. Dash, Mammogram classification using two dimensional discrete wavelet transform and gray-level co-occurrence matrix for detection of breast cancer. Neurocomputing, 154:114, 2015.

12. H. Zhang, R. Wu, T. Yuan, Z. Jiang, S. Huang, J. Wu, J. Hua, Z. Niu, and D. Ji "DE-Ada*: A novel model for breast mass classification using crossmodal pathological semantic mining and organic integration of multifeature fusions," Inf. Sci., vol. 539, pp. 461486, May 2020.

13. W. Xie W,Y. Li,Y. Ma, Breast mass classification in digital mammography based on extreme learning machine. Neurocomputing, 173: 930-941, 2016.

14. P. Qiong. An Image Segmentation Algorithm Research Based on Region Growth. Journal of Software Engineering, 9: 673-679, 2015.

15. A.Allaoui, M. Merzougui, M. Nasri, M. EL Hitmy and H. Ouariachi. Evolutionary Image Segmentation by Pixel Classification Application to Medical Images. IJCIIS International Journal of Computational Intelligence and Information Security, Vol. 2, No. 3 pp. 12- 24. March 2011.

16. J. Fan, G. Zeng, Mathurin Body, Mohand-Said Hacid. Seeded region growing: an extensive and comparative study. Pattern Recognition Letters 26, pp.11391156, 2005.

17. F. Besbes, N. Gargouri, A. Damak, W. Feki, D. Sellami, H. Fourati, Z. Mnif, and H. Besbes, "A Novel 3-D-CADx for Breast and Lesion Segmentation of Axial Breast DCE-MRI," Journal of Testing and Evaluation 49. Published ahead of print, 15 May 2020.

18. L. Wang, K. Filippatos, O. Friman, and H. K. Hahn, Fully Automated Segmentation of the Pectoralis Muscle Boundary in Breast MR Images, in Proceedings SPIE 7963, Medical Imaging 2011: Computer-Aided Diagnosis, ed. R. M. Summers and B. van Ginneken, 796309, 2011.

19. Ng KH, Muttarak M, Advances in mammography have improved early detection of breast cancer. J HK Coll Radiol 6:126131, 2003.

20. N. Gargouri, A. Dammak Masmoudi, D. Sellami, R. Abid, "A New GLLD Operator for Mass Detection in Digital Mammograms", International Journal of Biomedical Imaging, vol. 2012, Article ID 765649, 13 pages, 2012.

21. M. Zouari Mehdi, N. Gargouri, A. Damak, D. Sellami," A Textural Wavelet Quantization approach for an efficient breast microcalcifcation's detection", Multim. Tools Appl. 79(33-34): 24911-24927 (2020).

22. A. Damak Masmoudi, N. Gargouri, D. Sellami, R. Abid," LBPV descriptors-based automatic ACR/BIRADS classification approach", EURASIP J. Image Video Process. 2013: 19 (2013).

23. N. Gargouri, A. Dammak Masmoudi, D. Sellami and R. Abid, "New developments in the diagnostic procedures to reduce prospective biopsies breast," 2015 International Conference on Advances in Biomedical Engineering (ICABME), 2015, pp. 205-208, doi: 10.1109/ICABME.2015.7323288.

24. M. Felsberg and G. Sommer, The monogenic signal, IEEE Trans. SP, vol. 49, pp. 3136-3144, 2001.

25. O. Fleischmann, 2D signal analysis by generalized Hilbert transforms, Thesis, University of Kiel, 2008.

26. T Ojala, M Pietikainen, T Maenpaa, Multiresolution grayscale and rotation invariant texture classification with local binary patterns, IEEE Trans. PAMI, vol. 24, pp. 971-987, 2002.

27. L. Zhang, L. Zhang, Z. Guo and D. Zhang, "MonogenicLBP: A new approach for rotation invariant texture classification," 2010 IEEE International Conference on Image Processing, Hong Kong, pp. 2677-2680, 2010.
28. N. Gargouri Ben Ayed, A. Dammak Masmoudi, D. Sellami and R. Abid, "New developments in the diagnostic procedures to reduce prospective biopsies breast," 2015 International Conference on Advances in Biomedical Engineering (ICABME), 2015, pp. 205-208, doi: 10.1109/ICABME.2015.7323288.

29. R. Mokni, H. Drira, M. Kherallah, Fusing Multi-Techniques based on LDA-CCA and their Application in Palmprint Identification System. In Proceedings of the 2017 IEEE/ACS 14th International Conference on Computer Systems and Applications (AICCSA) (pp. 350357), 2017.

30. V.Vapnick, :"The nature of statistical learning theory", Springer-verlog, New-York, 1995.

31. T.Hastie, R. Tibshirani and J. Friedman, :"The elements of statistical learning: data mining, inference and prediction", Springer, 2009.

32. D.E. Rumelhart, G.E. Hinton, R.J. Williams:" Learning internal representations by error propagation, Parallel Distributed Processing". vol. 1, MIT Press, Cambridge, MA, pp. 318-362, 1986.

33. D. K. M. Heath and K. W. Bowyer:"Current status of the digital database for screening mammography". In em Proc. Proceedings of the fourth international workshop on digital mammography.

34. Zhu, Lei et al. Landmark Classification With Hierarchical Multi-Modal Exemplar Feature, IEEE Transactions on Multimedia 17, 981-993, 2015.

35. M Chen, Y Hao, K Lin, Z Yuan, L Hu, Label-less learning for traffic control in an edge network, IEEE Network 32 (6), $8-14,2018$.

36. W. Ayadi, W. Elhamzi,I. Charfi et al, Deep CNN for Brain Tumor Classification, Neural Process Lett 53, 671-700, 2021.

37. T. Ahonen, J. Matas, C. He, M. Pietikainen: "Rotation invariant image description with local binary pattern histogram Fourier features". Scandinavian Conference on Image Analysis, Lecture Notes in Computer Science, Springer, Berlin. vol. 5575, pp. 61-70, 2009. 